\title{
Aerial View of the Association between m6A- Related LncRNAs and Clinicopathological Characteristics of Pancreatic Cancer
}

\section{Bowen Huang}

Peking Union Medical College Hospital

Jun Lu

Peking Union Medical College Hospital

Dong Liu

Jinan University

\section{Wenyan Gao}

Cancer Hospital Chinese Academy of Medical Sciences

\section{Li Zhou}

Peking Union Medical College Hospital

\section{Feng Tian}

Peking Union Medical College Hospital

\section{Yizhi Wang}

Peking Union Medical College Hospital

\section{Bolun Jiang}

Peking Union Medical College Hospital

\section{Mingjie Luo}

Peking Union Medical College Hospital

\section{Chengxi Liu}

Peking Union Medical College Hospital

Jianzhou Liu

Peking Union Medical College Hospital

\section{Ziyu Xun}

Peking Union Medical College Hospital

\section{Congyong Xie}

Jinan University

Junchao Guo ( $\nabla$ gjcpumch@sohu.com )

Peking Union Medical College Hospital https://orcid.org/0000-0002-1174-924X 
Keywords: pancreatic cancer, m6A, IncRNA, prognostic model, clinicopathological characteristics

Posted Date: April 15th, 2021

DOI: https://doi.org/10.21203/rs.3.rs-370999/v1

License: (c) (1) This work is licensed under a Creative Commons Attribution 4.0 International License. Read Full License

Version of Record: A version of this preprint was published at Frontiers in Oncology on January 3rd, 2022. See the published version at https://doi.org/10.3389/fonc.2021.812785. 


\section{Abstract \\ Background}

There have been few reports on how long non-coding RNA (IncRNA) under the regulation of N6methyladenosine (m6A) modification influences pancreatic cancer progression. In our study, the association between m6A-related IncRNAs and pancreatic ductal adenocarcinoma (PDAC) was comprehensively described for the first time based on the construction of a IncRNAs prognostic model.

\section{Methods}

The IncRNAs expression level and the prognostic value were investigated in 440 PDAC patients and 171 normal tissues from Genotype-Tissue Expression (GTEx), The Cancer Genome Atlas (TCGA), and International Cancer Genome Consortium (ICGC) databases. We implemented Pearson correlation analysis to explore the m6A-related IncRNAs, univariate Cox regression and Kaplan-Meier (K-M) methods were performed to screen the critical IncRNAs in PDAC patients. Then we used bioinformatic analysis and statistical analysis to illustrate the association between m6A-related IncRNAs and pancreatic cancer.

\section{Results}

Seven prognostic m6A-related IncRNAs were identified as prognostic IncRNAs, and they were inputted in the Least Absolute Shrinkage and Selection Operator (LASSO) Cox regression to establish an m6A-related IncRNAs prognostic model in the TCGA database. Each patient has calculated a risk score and divided into low-risk and high-risk subgroups by the median value in two cohorts. Moreover, the model showed a robust prognostic ability in the stratification analysis of different risk subgroups, pathological grades, and recurrence events. The Cox regression demonstrated that the risk classification was an independent prognostic predictor. We established a competing endogenous RNA (ceRNA) network based on seven pivotal IncRNAs and twenty-six m6A regulators. Enrichment analysis indicated that malignancyassociated biological function and signaling pathways were enriched in the high-risk subgroup and $\mathrm{m} 6 \mathrm{~A}-$ related IncRNAs target mRNAs. We have even identified small molecule drugs that may affect the progression of pancreatic cancer.

\section{Conclusions}

In conclusion, we provide the first comprehensive aerial view between m6A-related IncRNAs and pancreatic cancer's clinicopathological characteristics.

\section{Background}


Of all the primary human cancers, pancreatic cancer has the worst prognosis. In the United States, approximately 57,600 people are diagnosed with pancreatic cancer each year, and 47,000 people die from this disease, ranking as the third leading cause of cancer death after lung cancer and colorectal cancer[1], with a 5 -year survival rate of $6 \%[2]$. After decades of fighting pancreatic cancer, surgical resection remains the only possible cure. Unfortunately, due to the late onset of clinical symptoms in pancreatic cancer patients, only $15 \%-20 \%$ of patients have the opportunity to undergo pancreatic resection, and the postoperative 5-year survival rate is only $18 \%[3]$. Patients who can't receive surgical treatment also can't benefit from chemical drugs, possibly because most of the patients with chemotherapy already have locally advanced or metastatic nidus. This is also the difficulty in diagnosing pancreatic cancer, often delayed due to the early stage's lack of symptoms. Therefore, it is imperative to have sensitive and accurate molecular markers in the early diagnosis, prognosis judgment, and treatment strategy selection of pancreatic cancer.

Most studies have suggested that m6A methylation, one of the most common RNA modifications, can affect the complexity of cancer progression by regulating biological functions related to cancer. M6A modification of noncoding RNAs regulates cleavage, transport, stability, and degradation [4]. The m6A regulators can be divided into three types: writers (methyltransferases), readers (signal transducers), and erasers (demethylases)[5]. Recent research had demonstrated that m6A modification could regulate tumorigenesis and progression in pancreatic cancer. For instance, The writer METTL3 promotes pancreatic cancer cell proliferation, invasion, chemoresistance, and radioresistance[6, 7]. The upregulation of reader HNRNPC was associated with rs7495G, which confer a higher risk of PDAC through a miRNA-mediated manner[8]. The eraser ALKBH5 prevents pancreatic cancer progression by posttranscriptional activation of PER1 through m6A abolishment, decreasing WIF-1 RNA methylation and mediating Wnt signaling $[9,10]$.

It's well known that IncRNAs' abnormal expression is closely connected with the degree of tumor malignancy. Very little research had found that m6A modification can affect the progression of pancreatic cancer by interfering with the expression of IncRNAs so far[11, 12]. Our team hoped to identify the prognostic significance of m6A-related IncRNAs by bioinformatics and statistical analysis of data from patients with PDAC based on GTEx, TCGA, and ICGC databases. Our program will find out the m6Arelated IncRNAs which had prognostic value in all databases PDAC patients. Furthermore, we constructed an m6A-related IncRNAs prognostic model to predict the overall survival of PDAC patients. Meanwhile, the stratified analysis was carried out with PDAC patients in different risk and clinicopathological subgroups, categorized based on the IncRNAs prognostic model. Furthermore, a ceRNA network was built to search the target miRNAs and m6A regulators of these m6A-related prognostic IncRNAs. Ultimately, we identified small molecule drugs that may interfere with pancreatic cancer progression by targeting $\mathrm{mRNA}$ expression levels. In a word, we have drawn a bird's eye view of the relationship between m6A-related IncRNAs and pancreatic cancer.

\section{Materials And Methods}


tabases and m6A-Related Genes

We merge the GTEx and TCGA databases as the training set, Fragments Per Kilobase of transcript per Million mapped reads (FPKM) normalized RNA-seq and the corresponding clinicopathological data were acquired from the University of California, Santa Cruz (UCSC) website (https://xenabrowser.net/datapages/). To obtain an ICGC validation set, we downloaded standardized RNA-seq data and related clinicopathological profiles from the ICGC website (https://daco.icgc.org/). We obtained a GTEx-TCGA training set involving 178 patients and 171 normal samples and an ICGC-CA validation dataset involving 262 patients.

In order to include all m6a-related genes that have been experimentally confirmed as much as possible, we searched PubMed for all literature associated with m6A modification. Several reviews that comprehensively summarized all the m6A regulatory genes were adopted[5, 13-16]. Finally, twenty-six genes were included in subsequent studies, including ten writers, fourteen readers and two erasers (Table 1).

\section{Annotation of lncRNAs}

The lncRNA annotation file of Genome Reference Consortium Human Build 38 (GRCh38) release 102 was acquired from the Ensembl website (http://asia.ensembl.org/index.html) for annotation of the lncRNAs in the GTEx-TCGA and ICGC databases. Based on recognizing the genes' Ensemble IDs, We could identify the lncRNAs in the GTEx-TCGA and the ICGC databases.

\section{Bioinformatic Analysis}

The Pearson correlation analysis was applied to mining m6A-related lncRNAs. We defined the $\mid$ Pearson $\mathrm{R} \mid>0.6$ and $\mathrm{p}<0.001$ as the criteria to extract m6A-related lncRNAs. Then univariate Cox regression and Kaplan-Meier (K-M) analyses were implemented to filtrate the prognostic m6A-related lncRNAs in the two databases. We use the Venn diagram to extract the pivotal lncRNAs that can satisfy the screening of two databases and two methods. The correlational relationships among the m6A related-lncRNAs in PDAC were analyzed based on Spearman's correction coefficient calculation. Moreover, using the R package "glmnet" to conduct Least Absolute Shrinkage and Selection Operator (LASSO) Cox regression[17], we could establish an m6A-related lncRNA prognostic model for the pancreatic cancer patients. The risk score calculating equation is:

$$
\text { Riskscore }=\sum_{k=1}^{n} \text { Coef }_{k} \times x_{k}
$$

Which Coef $_{k}$ means the coefficients, $x_{k}$ is the FPKM value of each prognostic lncRNAs.

Risk scores were calculated for all PDAC patients involving in our project. Using the GTExTCGA cohort, Differentially Expressed Genes (DEG) in the high-risk subgroup PDAC 
patients in contrast to the low-risk subgroup were identified based on the standards of I $\log 2$ (fold change) $\mid>0.5$ and $\mathrm{p}<0.05$ using the R package "limma"[18]. The DEG of the tumor and normal tissues in different subgroups according to the criterion of | $\log 2$ (fold change) $\mid>2$ and $\mathrm{p}<0.01$ in GETx-TCGA databases were shown used by the R package "limma" too. The R packages "clusterProfiler" and "org.Hs.eg.db" were library to Gene Ontology (GO) and Kyoto Encyclopedia of Genes and Genomes (KEGG) Pathway analyses. Python language was employed to perform the prediction analysis of the target miRNAs of the m6A-related lncRNAs in the miRcode (http://www.mircode.org/) and Starbase (http://starbase.sysu.edu.cn/) databases. What's more, we searched for the target mRNAs of these miRNAs in miRDB (http://mirdb.org/), miRTarBase (http://mirtarbase.cuhk.edu.cn/php/index.php), and Starbase databases. The ceRNA network was plotted using the software of "Cytoscape"[19]. Lastly, we used the Connectivity Map (CMAP) database (https://portals.broadinstitute.org/cmap/) to find out the small molecule drugs that might be related to PDAC in accordance with the genes that were directly different between tumor and normal samples mentioned above[20].

\section{Statistical Analyses}

The K-M curves and the log-rank test were utilized to compare all genes' overall survival and extract the m6a-related prognostic lncRNAs. We further compare the low-risk and high-risk subgroups and clinicopathologic subgroups based on the prognostic lncRNAs model. The prognostic ability of the model for 1/3/5-year overall survival was evaluated by receiver operating characteristic (ROC) curves and the area under the curve (AUC) values[21]. The student's t-test was used to compare the risk scores between pairs of subgroups in the TCGA database based on the following clinicopathological features: age, gender, smoking, drinking, diabetes, pancreatitis, grade, stage, TNM classification, location, recurrence, outcome, new tumor, multi-malignancies. Univariate and multivariate Cox regression analyses were employed to assess the independent prognostic value of the m6A-related lncRNAs prognostic model regarding overall survival in two cohorts. All statistical data and figures were analyzed by R (version 4.0.3) and GraphPad Prism 8.0 to ensure aesthetics and editability. All statistical results with a p-value $<0.05$ were considered significant.

\section{Results}

1. Identification of m6A-Related LncRNAs in PDAC Patients

Firstly, using the downloaded profile from the "Ensembl" website, we identified 4441 IncRNAs in the GTExTCGA database and 14181 IncRNAs in the ICGC-CA database based on recognizing the Ensemble IDs of the genes for the following analysis. In addition, we extracted the expression matrixes of 26 m6A-related genes from the GTEx-TCGA and the ICGC-CA databases. A IncRNAs whose expression value was 
correlated with one of the 26 m6A-related genes, with | Pearson $R \mid>0.6$ and $p<0.001$ as the criterion, was defined as an m6A-related IncRNA. We obtained 762 IncRNAs significantly correlated with m6Arelated genes in the GTEx-TCGA database, while 1370 IncRNAs in the ICGC database. Combined with the survival information, univariate Cox regression and Log-rank test were executed to screen seven m6Arelated prognostic IncRNAs in both databases (Figure 1A).

\section{Establish the m6a-Related LncRNAs Prognostic Model in the TCGA Database}

All m6A-related IncRNAs showed positive co-expression, which presented an extensive synergy effect (Figure 1B, C). The IncRNA CASC19 and LINC02323 possessed the most significant correlation coefficient (0.68) in the GTEx-TCGA database, while the IncRNA ITGB1-DT and LINC02323, NRAV and PRECSIT had the largest correlation coefficient (0.68) in the ICGC database. It is worth mentioning that NRAV and PRECSIT also possessed a significant correlation coefficient (0.64) in the GTEX-TCGA database. In order to build the m6A-related IncRNAs prognostic model for forecasting the overall survival of PDAC patients, at the same time, avoid the expression overfitting possibility brought by high correlation, we performed a LASSO regression analysis based on the seven m6A-related prognostic IncRNAs in the TCGA cohort. Moreover, it generated the prognostic model containing seven m6A-related IncRNAs and each IncRNA coefficient (Figures 1D, E). For each patient in the TCGA database, a risk score was calculated based on the coefficient for each IncRNAs (Figure 1F). Patients in the TCGA training cohort were divided into lowrisk and high-risk subgroups based on the median value of risk scores. K-M curves demonstrated that PDAC patients with higher risk scores had worse outcomes (Figure 1G). The ROC curves illustrated that the IncRNAs prognostic model has an excellent predictive ability to predict overall survival in the TCGA training cohort (1-year AUC $=0.710$, 3-year $A U C=0.803,5$-year $A U C=0.887$; Figure 11). Risk score and survival status distributions are plotted in Figure $1 \mathrm{~K}$.

3. Validation of the LncRNAs Prognostic Model in the ICGC Database

To validate the IncRNAs prognostic model's predictive ability based on the TCGA training set, we calculated risk scores for patients in the ICGC cohort using the same equation. PDAC patients in the ICGC database were assigned to low-risk and high-risk subgroups according to the median risk score. The results were consistent with the TCGA database findings: PDAC patients with higher risk scores had lower overall survival rates and a shorter overall survival time in the ICGC dataset (Figure $1 \mathrm{H}$ ). The ROC curves also demonstrated that m6A-related IncRNAs prognostic model had a robust prognostic value for PDAC patients in the ICGC database (1-year AUC = 0.641, 3-year AUC = 0.698, 5-year AUC = 0.711; Figure 1J). Risk score and survival status distributions are shown in Figure $1 \mathrm{~L}$, and it showed that patients with higher risk scores had shorter overall survival time and more death status. These results showed that the IncRNAs prognostic model was a robust and stable overall survival predictive tool. 


\section{Prognostic Analysis of the Seven m6A-Related LncRNAs}

Univariate Cox regression analysis was employed to evaluate seven m6A-related IncRNAs in the prognostic model and their prognostic roles. The forest plot shows that all of them are risk factors with Hazard Ratio (HR) >1 in PDAC patients (Figure 2A). The K-M survival curves confirmed that higher expression of CASC19, ITGB1-DT, LINC01094, LINC02323, NRAV, PRECSIT, and UCA1 were associated with worse overall survival in the TCGA database (Figures 2B-H).

\section{Pathway and Process Enrichment Analysis of Different Risk Subgroups}

For investigating the potential biological process and pathway involving in the molecular heterogeneity between the low-risk and high-risk subgroups, we identified 1107 differential expression genes (DEGs) between the low-risk and high-risk subgroups in the TCGA cohort with the filter criteria |log2 (fold change) $\mid>0.5$ and $p<0.05$ (Figure 2 I). These DEGs were primarily enriched in these $G 0$ terms: the biological process (BP) included the leukocyte migration, epidermis development, cell junction assembly, skin development, positive regulation of cell adhesion, etc; the cell component (CC) contains cell-cell junction, collagen-containing extracellular matrix, apical part of cell, external side of plasma membrane, apical plasma membrane, etc; the molecular function (MF) included cell adhesion molecule binding, receptor ligand activity, actin binding, cadherin binding, cell adhesion mediator activity, etc (Figure 2J). The KEGG analyses revealed that 16 tumor characteristics were enriched in the high-risk subgroup, such as hematopoietic cell lineage, cell adhesion molecules, insulin secretion, malaria, axon guidance, etc (Figure 2K). These results may disclose some perspectives into the cellular biological effects related to the m6A-related IncRNAs prognostic model.

\section{Stratification Analysis of the m6A-Related LncRNAs Prognostic Model}

The heatmap exhibited that LINC01094, CASC19, LINC02323, PRECSIT, UCA1, ITGB1-DT, and NRAV were enriched in the high-risk subgroup, meanwhile, showed the association between each m6A-related IncRNAs expression and the clinicopathological features of PDAC patients (Figure 3A). We attempted to identify whether clinicopathological characteristics were connected with the risk score (Figure 3B-R). The results revealed that the PDAC patients with a higher risk score, the tumor issue have a worse pathological differentiation and showed a strictly increasing relationship $(P<0.05)$. Besides, we found that the PDAC patients with higher risk scores were also more likely to have tumor recurrence after treatment $(P<0.05)$. The patients with distant metastasis had the highest risk score, followed by the locoregional recurrence. The new primary tumor had the lowest risk score with only three samples. To better assess the m6A-related IncRNAs prognostic model's prognostic capacity, we carried out a stratification analysis to verify whether it remains its ability to forecast overall survival in various subgroups. According to the 
clinicopathological characteristics, we performed K-M curves for subgroups with each risk stratification of more than five persons. In contrast with lower risk score patients, higher risk PDAC patients had worse overall survival in both the pathological G2 and G3 grades, while there was no significant difference in the G1 stratification (Figure 4A-C). Likewise, we confirmed that the m6A-related IncRNAs prognostic model retained its capacity to predict overall survival for whether PDAC patients have recurrence events or not. Moreover, the further detailed stratification was performed, we detected that the PDAC patients with distant metastasis had shorter survival time, On the contrary, the locoregional recurrence patients had no significant difference (Figure 4D-G).

7. LncRNAs Prognostic Model Was an Independent Prognostic Factor for PDAC Patients

We used univariate and multivariate Cox analyses to assess whether the m6A-related IncRNAs prognostic model was an independent prognostic factor for patients with PDAC. Based on the data of PDAC patients in the TCGA database, univariate Cox analysis indicated that IncRNAs prognostic model was remarkably associated with overall survival [the hazard ratio (HR): $2.960,95 \%$ confidence interval (CI): 1.807-4.849, p $<0.001$; Figure $4 \mathrm{H}$ ] and multivariate Cox analysis further showed that IncRNAs prognostic model was an independent predictor of overall survival, with the $\mathrm{HR}(95 \% \mathrm{Cl})$ was $2.966(1.687-5.215)(p<0.001$, Figure $4 I)$. The same results were verified in the ICGC database with less clinicopathological characteristics abundance (Figure $4 \mathrm{~J}, \mathrm{~K}$ ). These results indicated that the m6A-related IncRNAs prognostic model might help clinical prognosis evaluation as an independent prognostic indicator.

\section{The Differential Expression Level of Each m6A-Related LncRNAs}

We analyzed the expression of each m6A-related IncRNAs in PDAC patients compared to normal pancreas tissues in the GTEx-TCGA database using the R package "limma." We generated boxplots for seven critical IncRNAs and observed a statistically significant increased expression in tumor samples for all m6A-related IncRNAs based on the Bayesian algorithm ( $p<0.001$, Figure $5 A-G)$.

\section{Construction of the ceRNA Network and Functional Enrichment Analysis}

To further understand how the critical IncRNAs act on N6-methyladenosine regulators by sponging miRNAs in PDAC patients, we constructed a ceRNA network to explore the mechanism of m6A-related IncRNAs. Six IncRNAs were extracted from the Starbase and miRcode databases, and 162 pairs of interactions between the six IncRNAs and 153 miRNAs were identified. Then we excavated three databases (Starbase, miRDB, and miRTarBase) to search target N6-methyladenosine regulator based on the 153 miRNAs and a total of 890 pairs of interactions between the 153 miRNAs and 26 m6A regulators were identified in all three databases. Finally, 6 IncRNAs, 153 miRNAs, and 26 m6A regulators were 
included in the ceRNA network (Figure 5H). Furthermore, there are 17288 mRNAs we extract from the three databases the 153 miRNAs target, and we affirmed 1145 DEGs from the GTEx-TCGA database with the filter criteria $\mid \log 2$ (fold change) $\mid>2$ and $p<0.01$. These DEGs were wielded to implemented functional enrichment analysis and we found that these genes were enriched in the BP included extracellular matrix organization, extracellular structure organization, neutrophil activation, etc; the CC contains collagen-containing extracellular matrix, cell-substrate junction, focal adhesion, etc; the MF included cell adhesion molecule binding, extracellular matrix structural constituent, glycosaminoglycan binding, etc (Figure 5I). KEGG analysis showed that 31 signaling pathways were enriched in pancreatic cancer, some of which had tumor characteristics, including protein digestion and absorption, ECMreceptor interaction, focal adhesion, etc (Figure 5J). These data may provide medical workers clues for finding the potential pathways of these m6A-related IncRNAs in PDAC.

\section{Exploration of Small Molecule Drugs Related to Pancreatic Cancer}

We put the 1145 DEGs mentioned above into the CMAP database for analysis. We set $P<0.01$,|mean| $>$ 0.6 as the filter criteria, and extract twelve small molecule drugs related to PDAC. The Thapsigargin, Adiphenine, Viomycin, and Nadolol negative control the m6A-related IncRNAs targeted mRNA expression. While the Mepacrine, Ellipticine, 8-azaguanine, DL-thiorphan, Proscillaridin, Trazodone, Bisacodyl, and Riboflavin positive regulated the targeted mRNA expression level (Table 2).

\section{Discussion}

A total of 440 PDAC tumor samples and 171 normal tissues from the GTEX-TCGA and ICGC cohorts were included in our study to exploit the prognostic significance of m6A-related IncRNAs. Seven m6A-related IncRNAs were confirmed to have prognostic value in both the TCGA and ICGC databases, and were used to establish an m6A-related IncRNAs prognostic model for predicting the overall survival of PDAC patients. Based on each cohort's median risk score, PDAC patients were divided into the low-risk and highrisk subgroups, and the high-risk group had worse clinical outcomes and enrichment of neoplasm characteristics and specific malignant-related pathways. The higher patients' risk score, the worse the pathological grade and the more recurrent events. Multivariate Cox regression analysis showed that the m6A-related IncRNAs prognostic model and initial treatment outcome were the independent risk factors for overall survival. Meanwhile, we found that seven IncRNAs were highly expressed in tumor samples, which is good for clinical workers to screen and diagnose PDAC patients. A ceRNA network consisted of 6 m6A-related IncRNAs, 153 miRNAs, and 26 m6A regulators to view this IncRNAs prognostic model's potential functions. Simultaneously, we did the enrichment analysis of the targeted mRNA to discover its potential biological function and signal pathway based on the m6A-related IncRNAs prognostic model. In the end, we founded that Thapsigargin, Adiphenine, Viomycin, and Nadolol may be the small molecular drug to cure pancreatic cancer through intervening in the expression of the m6A-related IncRNAs target 
mRNA. While the Mepacrine, Ellipticine, 8-azaguanine, DL-thiorphan, Proscillaridin, Trazodone, Bisacodyl, and Riboflavin maybe can be used to build a pancreatic cancer tumor experimental animal model.

Multiple projects have suggested that m6A modification might function as a regulator in oncogenicity, but how it acts in a IncRNA-dependent pattern during PDAC progression is still unclear. To date, m6A regulators can maintain the malignancy of PDAC by modifying specific IncRNAs has only been mentioned in a few articles. He et al. have founded ALKBH5 inhibits pancreatic cancer motility by demethylating IncRNA KCNK15-AS1[12]. The research may be the earliest experimental exploration of how IncRNA affects pancreatic cancer through m6A modification. Shortly after that, Hu et al. demonstrated that IncRNA DANCR targets IGF2BP2 through m6A modification, and IGF2BP2 and DANCR work together to promote cancer stemness-like properties and pancreatic cancer pathogenesis[11]. Meng et al. revealed N6-Methyladenosine was highly enriched within LINC00857 and enhanced its RNA stability. Meanwhile, LINC00857 modulates E2F3 expression by binding to miR-150-5p, ultimately promoting tumorigenesis in pancreatic cancer[22]. Studies had disclosed that m6A modification of IncRNAs could influence pancreatic cancer tumorigenesis, and IncRNAs might serve as competing endogenous RNAs, targeting m6A regulators and thereby influencing aggressive tumor progression. Based on the above considerations, we believe that IncRNAs participated in m6A modification, and we ought to pay more attention to the interactions and functions of IncRNAs and m6A modifications so as to identify prognostic markers and therapeutic targets of pancreatic cancer.

We identified seven m6A-related prognostic IncRNAs from three databases and 611 samples. The IncRNA CASC19 participated in developing pancreatic cancer with CASC19/miR-148b/E2F7 axis[23]. Multiple studies have indicated that UCA1 acts as a ceRNA in the development and progression of pancreatic cancer in multiple axes(24-26). LINC02323 sponged miR-1343-3p to upregulate the TGFBR1 expression and promote the epithelial-mesenchymal transition and metastasis in lung adenocarcinoma[27]. PRECSIT promotes the progression of cutaneous squamous cell carcinoma via STAT3 signaling[28]. LINC01094 facilitates clear cell renal cell carcinoma radioresistance by targeting the miR577/CHEK2/FOXM1 axis[29]. NRAV has a certain suggestive effect in predicting the prognosis of hepatocellular carcinoma[30]. ITGB1-DT is a brand new IncRNA, and no relevant research has been found so far. Several of the seven IncRNAs were reported to be associated with oncogenesis, but there have been few reports regarding pancreatic cancer and no reports on how the IncRNAs interact with the m6A regulator so far. Our study identified the seven pivotal m6A-related prognostic IncRNAs, thereby providing insights into their potential roles in PDAC tumorigenesis and progression.

Although we built a robust and reliable model, there were several limitations in our study. The K-M curves of patients with $\mathrm{G} 1$ and $\mathrm{G} 3$ pathologic grades intersect between half and one year because most patients received surgical treatment. In the short term, patients who receive surgical treatment are at risk of complications that lead to death after surgery, resulting in a bias. In the long term, low-risk patients had longer survival times across all pathologic stratifications. Another hand, Pancreatic cancer patients are scarce, and the lack of data to analyze may also lead to bias. Moreover, the IncRNAs' role and their 
interactions with m6A regulators should be confirmed through experiments, which is the next step for our team to explore.

There are few studies on how IncRNAs affect the progression of pancreatic cancer through the m6A modification. Our project has filled the gap in predicting clinical prognosis based on m6A-related IncRNAs. We established the world's first m6A-related IncRNAs prognostic model for pancreatic cancer and confirmed its robust predictive ability in multiple databases. Secondly, we analyzed the relationship between risk score and abundant clinicopathological characteristics based on the prognostic model and verified that IncRNAs prognostic model was an independent prognostic factor for PDAC patients, which has not been studied in this field so far. Finally, we analyzed the possible biological mechanism and signaling pathway of the pivotal m6A-related IncRNAs, and found out its ceRNA network between IncRNAs and m6A-regulators through miRNAs. By targeting mRNA, we could find out small molecule drugs that can be used to treat or promote pancreatic cancer, closely combining theory with clinical practice and providing more attractive clues for researchers studying pancreatic cancer.

\section{Conclusion}

We developed a robust m6A-related IncRNA prognostic model for clinical workers to predict PDAC overall survival. Moreover, the stratification analysis demonstrated the worse pathological grade and the more recurrent events were associated with the higher risk score. The enrichment analysis indicated that malignancy-associated biological function and signaling pathways were enriched in the high-risk subgroup and m6A-related IncRNAs target mRNAs. Besides, the small molecule drugs that may affect the progression of PDAC were identified. In conclusion, we provide the first comprehensive aerial view between m6A-related IncRNAs and pancreatic cancer's clinicopathological characteristics.

\section{Declarations}

\section{Acknowledgements}

Not applicable.

\section{Consent for publication}

Not applicable.

\section{Authors' contributions}

Conceptualization, BWH and JCG; methodology, BWH and JL; software, BWH, DL, and WYG; validation, LZ and FT; formal analysis, YZW and BLJ; investigation, MJL, CXL and JZL; data curation, ZYX and CYX; 
writing-original draft preparation, $\mathrm{BWH}$ and JL; writing-review and editing, $\mathrm{BWH}, \mathrm{DL}$, and WYG; project administration, BWH and JCG; funding acquisition, JCG. All authors have read and agreed to the published version of the manuscript.

\section{Author details}

1. Department of General Surgery, State Key Laboratory of Complex Severe and Rare Diseases, Peking Union Medical College Hospital, Chinese Academy of Medical Sciences and Peking Union Medical College, Beijing, CN 100730.

2. Jinan University, Guangzhou, CN 510632.

3. State Key Laboratory of Molecular Oncology, National Cancer Center/National Clinical Research Center for Cancer/Cancer Hospital, Chinese Academy of Medical Sciences and Peking Union Medical College, Beijing, CN 100021.

\section{Competing interests}

The authors declare that they have no competing interests.

\section{Availability of data and materials}

The datasets used and/or analyzed during the current study are available from the corresponding author upon reasonable request.

\section{Ethics approval and consent to participate}

No ethical approval nor informed consent was required in this study due to the public-availability of the data used.

\section{Funding}

This study was supported by the National Natural Science Foundation of China (Grant No. 81972324), the Chinese Academy of Medical Sciences Innovation Fund for Medical Sciences (Grant No. 2016-I2M-3019), and the non-profit Central Research 
Institute Fund of Chinese Academy of Medical Sciences (Grant No. 2018PT32014).

\section{References}

1. Siegel RL, Miller KD, Jemal A. Cancer statistics, 2020. Cancer J Clin. 2020;70(1):7-30.

2. Kamisawa T, Wood LD, Itoi T, Takaori K. Pancreatic cancer. The Lancet. 2016;388(10039):73-85.

3. Mayo SC, Nathan H, Cameron JL, Olino K, Edil BH, Herman JM, Hirose K, Schulick RD, Choti MA, Wolfgang $\mathrm{CL}$, et al. Conditional survival in patients with pancreatic ductal adenocarcinoma resected with curative intent. CANCER-AM CANCER SOC. 2012;118(10):2674-81.

4. Ma S, Chen C, Ji X, Liu J, Zhou Q, Wang G, Yuan W, Kan Q, Sun Z. The interplay between m6A RNA methylation and noncoding RNA in cancer. Journal of hematology oncology. 2019;12(1):121.

5. He L, Li H, Wu A, Peng Y, Shu G, Yin G. Functions of N6-methyladenosine and its role in cancer. MOL CANCER. 2019;18(1):176.

6. Xia T, Wu X, Cao M, Zhang P, Shi G, Zhang J, Lu Z, Wu P, Cai B, Miao Y, et al. The RNA m6A methyltransferase METTL3 promotes pancreatic cancer cell proliferation and invasion. Pathology Research Practice. 2019;215(11):152666.

7. Taketo K, Konno M, Asai A, Koseki J, Toratani M, Satoh T, Doki Y, Mori M, Ishii H, Ogawa K. The epitranscriptome m6A writer METTL3 promotes chemo- and radioresistance in pancreatic cancer cells. INT J ONCOL. 2018;52(2):621-9.

8. Ying P, Li Y, Yang N, Wang X, Wang H, He H, Li B, Peng X, Zou D, Zhu Y, et al. Identification of genetic variants in m6A modification genes associated with pancreatic cancer risk in the Chinese population. ARCH TOXICOL. 2021;95(3):1117-28.

9. Guo X, Li K, Jiang W, Hu Y, Xiao W, Huang Y, Feng Y, Pan Q, Wan R. RNA demethylase ALKBH5 prevents pancreatic cancer progression by posttranscriptional activation of PER1 in an m6AYTHDF2-dependent manner. MOL CANCER 2020, 19(1).

10. Tang $B$, Yang $Y$, Kang M, Wang $Y$, Wang $Y, B i$ Y, He S, Shimamoto F. m6A demethylase ALKBH5 inhibits pancreatic cancer tumorigenesis by decreasing WIF-1 RNA methylation and mediating Wnt signaling. MOL CANCER 2020, 19(1).

11. Hu X, Peng W, Zhou H, Jiang J, Zhou X, Huang D, Mo Y, Yang L. IGF2BP2 regulates DANCR by serving as an N6-methyladenosine reader. Cell Death Differentiation. 2020;27(6):1782-94.

12. He Y, Hu H, Wang Y, Yuan H, Lu Z, Wu P, Liu D, Tian L, Yin J, Jiang K, et al. ALKBH5 Inhibits Pancreatic Cancer Motility by Decreasing Long Non-Coding RNA KCNK15-AS1 Methylation. CELL PHYSIOL BIOCHEM. 2018;48(2):838-46.

13. Huang $\mathrm{H}$, Weng $\mathrm{H}$, Chen J. $\mathrm{m}(6) \mathrm{A}$ Modification in Coding and Non-coding RNAs: Roles and Therapeutic Implications in Cancer. CANCER CELL. 2020;37(3):270-88.

14. Zaccara S, Ries RJ, Jaffrey SR. Reading, writing and erasing mRNA methylation. Nat Rev Mol Cell Biol. 2019;20(10):608-24. 
15. Huang $\mathrm{H}$, Weng $\mathrm{H}$, Chen $\mathrm{J}$. The Biogenesis and Precise Control of RNA m(6)A Methylation. TRENDS GENET. 2020;36(1):44-52.

16. Livneh I, Moshitch-Moshkovitz S, Amariglio N, Rechavi G, Dominissini D. The m(6)A epitranscriptome: transcriptome plasticity in brain development and function. NAT REV NEUROSCl. 2020;21(1):36-51.

17. Friedman J, Hastie T, Tibshirani R. Regularization Paths for Generalized Linear Models via Coordinate Descent. J STAT SOFTW. 2010;33(1):1-22.

18. Ritchie ME, Phipson B, Wu D, Hu Y, Law CW, Shi W, Smyth GK. limma powers differential expression analyses for RNA-sequencing and microarray studies. NUCLEIC ACIDS RES. 2015;43(7):e47.

19. Shannon P, Markiel A, Ozier O, Baliga NS, Wang JT, Ramage D, Amin N, Schwikowski B, Ideker T. Cytoscape: a software environment for integrated models of biomolecular interaction networks. GENOME RES. 2003;13(11):2498-504.

20. Lamb J, Crawford ED, Peck D, Modell JW, Blat IC, Wrobel MJ, Lerner J, Brunet J, Subramanian A, Ross $\mathrm{KN}$, et al. The Connectivity Map: Using Gene-Expression Signatures to Connect Small Molecules, Genes, and Disease. Science. 2006;313(5795):1929-35.

21. Blanche P, Dartigues J, Jacqmin-Gadda H. Estimating and comparing time-dependent areas under receiver operating characteristic curves for censored event times with competing risks. STAT MED. 2013;32(30):5381-97.

22. Meng X, Deng Y, He S, Niu L, Zhu H. m(6)A-Mediated Upregulation of LINC00857 Promotes Pancreatic Cancer Tumorigenesis by Regulating the miR-150-5p/E2F3 Axis. FRONT ONCOL. 2021;11:629947.

23. Lu T, Wei GH, Wang J, Shen J. LncRNA CASC19 contributed to the progression of pancreatic cancer through modulating miR-148b/E2F7 axis. Eur Rev Med Pharmacol Sci. 2020;24(20):10462-71.

24. Gong J, Lu X, Xu J, Xiong W, Zhang H, Yu X. Coexpression of UCA1 andITGA2in pancreatic cancer cells target the expression of miR-107 through focal adhesion pathway. J CELL PHYSIOL. 2019;234(8):12884-96.

25. Guo Z, Wang X, Yang Y, Chen W, Zhang K, Teng B, Huang C, Zhao Q, Qiu Z. Hypoxic Tumor-Derived Exosomal Long Noncoding RNA UCA1 Promotes Angiogenesis via miR-96-5p/AMOTL2 in Pancreatic Cancer. Molecular therapy Nucleic acids. 2020;22:179-95.

26. Liu Y, Feng W, Gu S, Wang H, Zhang Y, Chen W, Xu W, Lin C, Gong A, Xu M. The UCA1/KRAS axis promotes human pancreatic ductal adenocarcinoma stem cell properties and tumor growth. AM J CANCER RES. 2019;9(3):496-510.

27. Zhang X, Du L, Han J, Li X, Wang H, Zheng G, Wang Y, Yang Y, Hu Y, Wang C. Novel long non-coding RNA LINC02323 promotes epithelial-mesenchymal transition and metastasis via sponging miR1343-3p in lung adenocarcinoma. THORAC CANCER. 2020;11(9):2506-16.

28. Piipponen M, Nissinen L, Riihila P, Farshchian M, Kallajoki M, Peltonen J, Peltonen S, Kahari VM. p53Regulated Long Noncoding RNA PRECSIT Promotes Progression of Cutaneous Squamous Cell Carcinoma via STAT3 Signaling. AM J PATHOL. 2020;190(2):503-17. 
29. Jiang Y, Li W, Yan Y, Yao X, Gu W, Zhang H. LINC01094 triggers radio-resistance in clear cell renal cell carcinoma via miR-577/CHEK2/FOXM1 axis. CANCER CELL INT 2020, 20(1).

30. Xu Q, Wang Y, Huang W. Identification of immune-related IncRNA signature for predicting immune checkpoint blockade and prognosis in hepatocellular carcinoma. INT IMMUNOPHARMACOL. 2021;92:107333.

\section{Tables}

Table 1. The list of the 26 m6A-related methylation regulative factors from publications.

\begin{tabular}{llll}
\hline m6a Type & Regulator & Gene Synonyms & Ensembl ID \\
\hline Writer & METTL3 & M6A, MT-A70, Spo8 & ENSG00000165819 \\
& METTL14 & KIAA1627 & ENSG00000145388 \\
& METTL16 & METT10D, MGC3329 & ENSG00000127804 \\
& ZCCHC4 & FLJ23024, HSPC052, ZGRF4 & ENSG00000168228 \\
& WTAP & KIAA0105, MGC3925, Mum2 & ENSG00000146457 \\
& VIRMA & DKFZP434116, KIAA1429, fSAP121 & ENSG00000164944 \\
& RBM15 & OTT, OTT1 & ENSG00000162775 \\
& RBM15B & HUMAGCGB, OTT3 & ENSG00000259956 \\
& ZC3H13 & DKFZp434D1812, KIAA0853, Xio & ENSG00000123200 \\
CBLL1 & FLJ23109, HAKAI, RNF188 & ENSG00000105879 \\
Reader & YTHDF1 & C20orf21, FLJ20391 & ENSG00000149658 \\
& YTHDF2 & CAHL, HGRG8, NY-REN-2 & ENSG00000198492 \\
& YTHDF3 & FLJ31657 & ENSG00000185728 \\
& YTHDC1 & KIAA1966, YT521, YT521-B & ENSG00000083896 \\
& YTHDC2 & DKFZp564A186, FL10053, FLJ2194 & ENSG00000047188 \\
& IGF2BP1 & IMP-1 & ENSG00000159217 \\
& IGF2BP2 & IMP-2 & ENSG00000073792 \\
& IGF2BP3 & CT98, IMP-3, IMP3 & ENSG00000136231 \\
& HNRNPC & HNRPC & ENSG00000092199 \\
& RBMX & HNRNPG, RNMX, hnRNP-G & ENSG00000147274 \\
& HNRNPA2B1 & HNRPA2B1 & ENSG00000122566 \\
& EIF3A & EIF3, KIAA0139 & ENSG00000107581 \\
& FMR1 & FMRP, FRAXA, MGC87458, POF, POF1 & ENSG00000102081 \\
& PRRC2A & BAT2, D6S51E, G2 & ENSG00000204469 \\
FTO & ALKBH9, KIAA1752, MGC5149 & ENSG00000140718 \\
& FLKBH5 & FLJ20308, OFOXD1 & ENSG00000091542 \\
\hline
\end{tabular}

Table 2. CMAP was used to explore the potential drug to cure PDAC according to the expression level of targeted mRNA in the ceRNA network in the GTEx-TCGA database $(\mathrm{P}<$ $0.01, \mid$ mean $\mid>0.6)$. 


\begin{tabular}{llllll}
\hline Cmap name & mean & $\mathrm{n}$ & enrichment & $\mathrm{p}$ & specificity \\
\hline Thapsigargin & -0.893 & 3 & -0.99 & 0 & 0.0065 \\
Adiphenine & -0.71 & 5 & -0.82 & 0.00046 & 0.0645 \\
Viomycin & -0.663 & 4 & -0.854 & 0.00084 & 0.0576 \\
Nadolol & -0.627 & 4 & -0.867 & 0.00062 & 0.0154 \\
Riboflavin & 0.639 & 4 & 0.776 & 0.00471 & 0.0248 \\
Bisacodyl & 0.702 & 4 & 0.851 & 0.00072 & 0.0053 \\
Trazodone & 0.742 & 3 & 0.894 & 0.00232 & 0.0224 \\
Proscillaridin & 0.752 & 3 & 0.917 & 0.00118 & 0.0545 \\
DL-thiorphan & 0.756 & 2 & 0.928 & 0.00984 & 0.0294 \\
8-azaguanine & 0.761 & 4 & 0.877 & 0.00032 & 0.0355 \\
Ellipticine & 0.773 & 4 & 0.813 & 0.00235 & 0.0774 \\
Mepacrine & 0.8 & 2 & 0.977 & 0.00087 & 0.0052 \\
\hline
\end{tabular}

Figures 
A

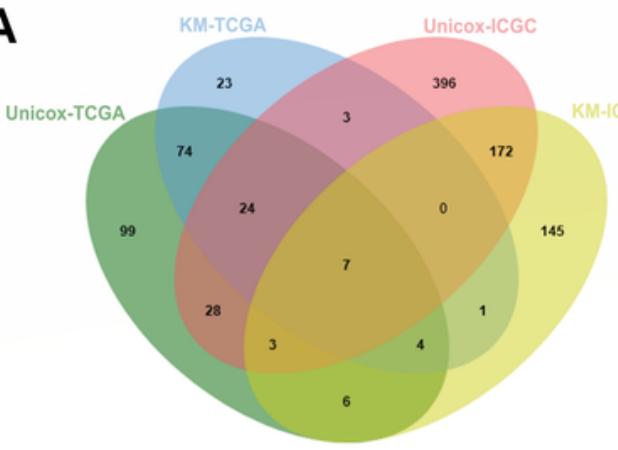

B

D

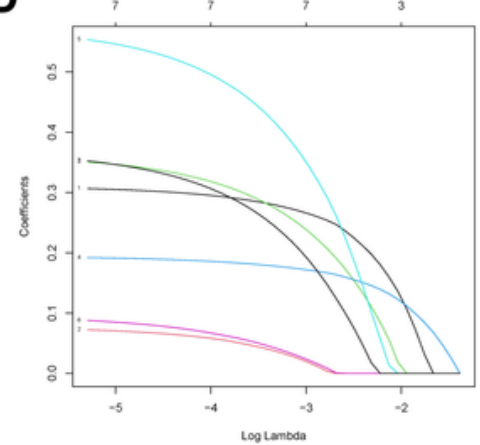

E

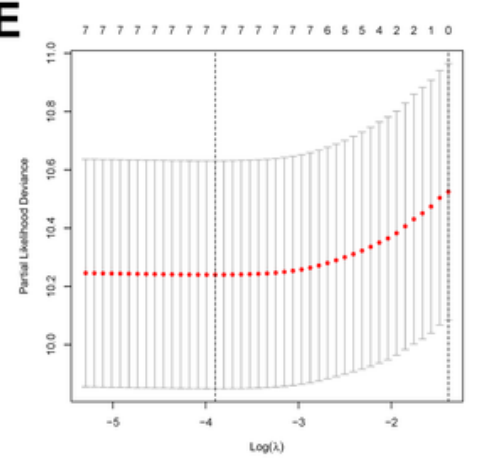

C

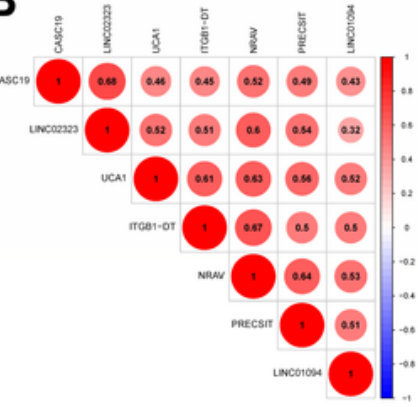

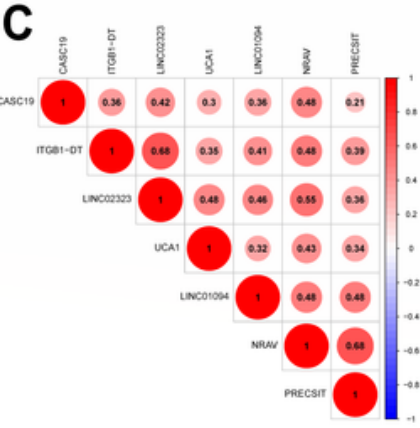

$\mathbf{F}$

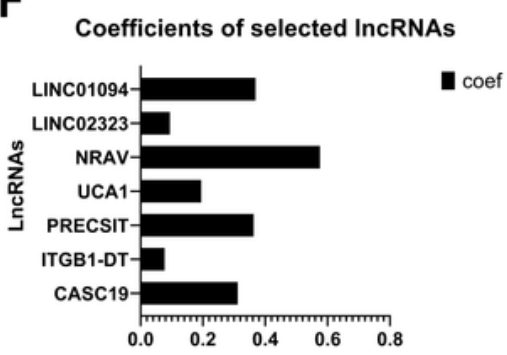

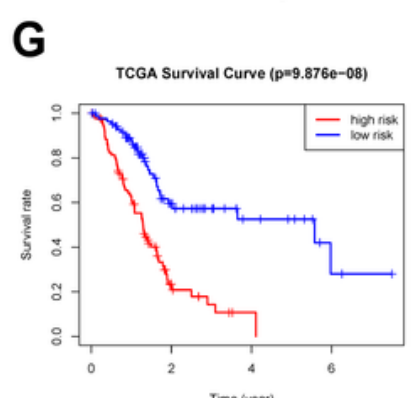

K

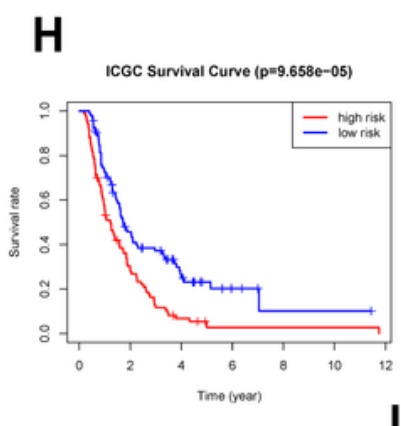

I

$\mathrm{L}$
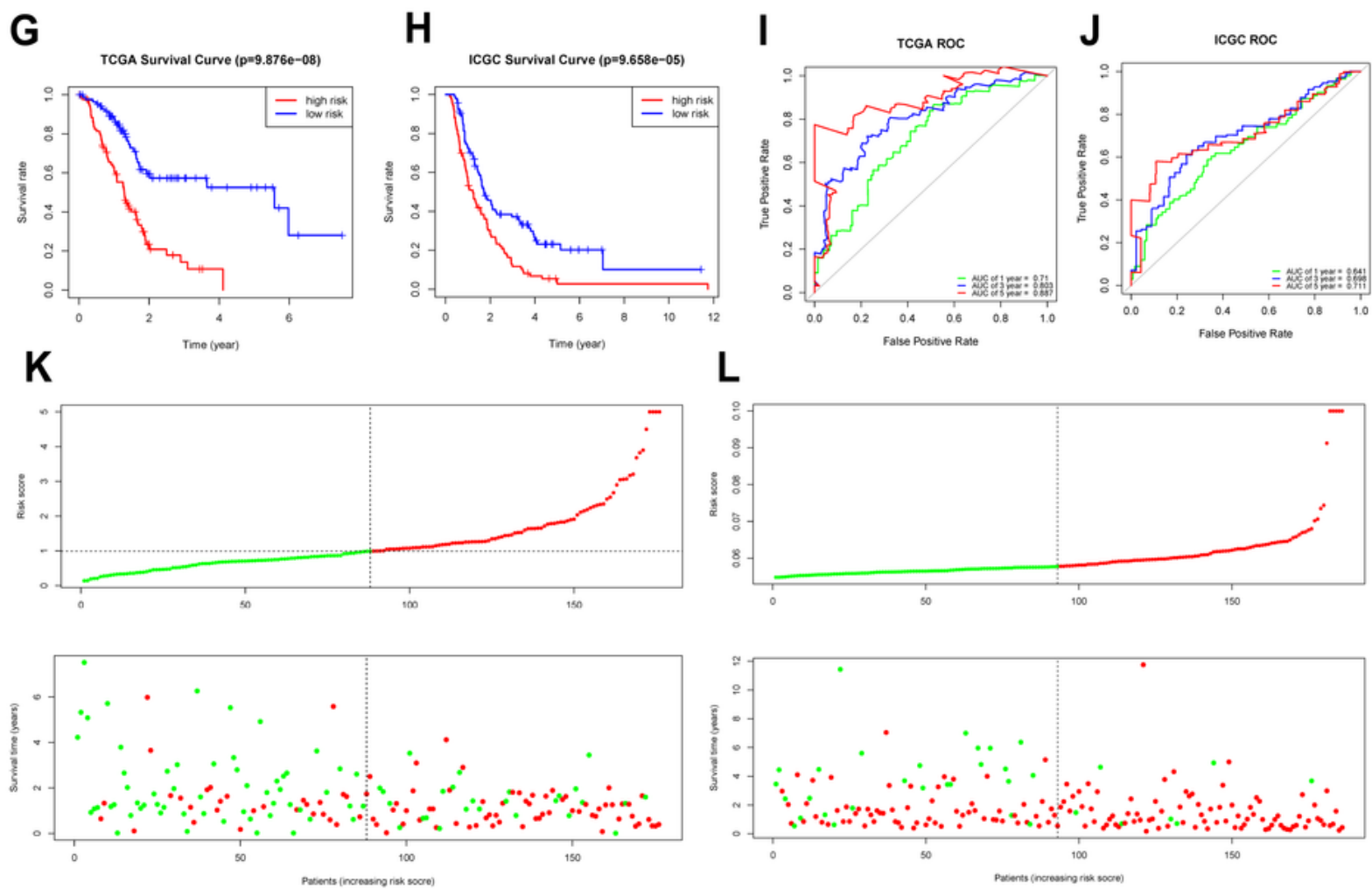

\section{Figure 1}

(A) The critical prognostic IncRNA-signatures in GTEx-TCGA and ICGC databases were screened by Venn's diagram. (B-C) The correlation heatmap of the GTEx-TCGA database (B) and ICGC database (C). (D-F) Used the LASSO regression to calculate the minimum value of lambda (D, E) and coefficients (F). (G, H) K$M$ curves showed that the high-risk subgroup had worse overall survival than the low-risk subgroup in TCGA (G) and ICGC (H) databases. $(\mathrm{I}, \mathrm{J})$ ROC curves of IncRNA-signatures for predicting the $1 / 3 / 5$-year 
survival in the TCGA (I) and ICGC $(J)$ databases. $(K, L)$ Distributions of risk scores and survival status of PDAC patients in the TCGA $(K)$ and ICGC $(L)$ databases.
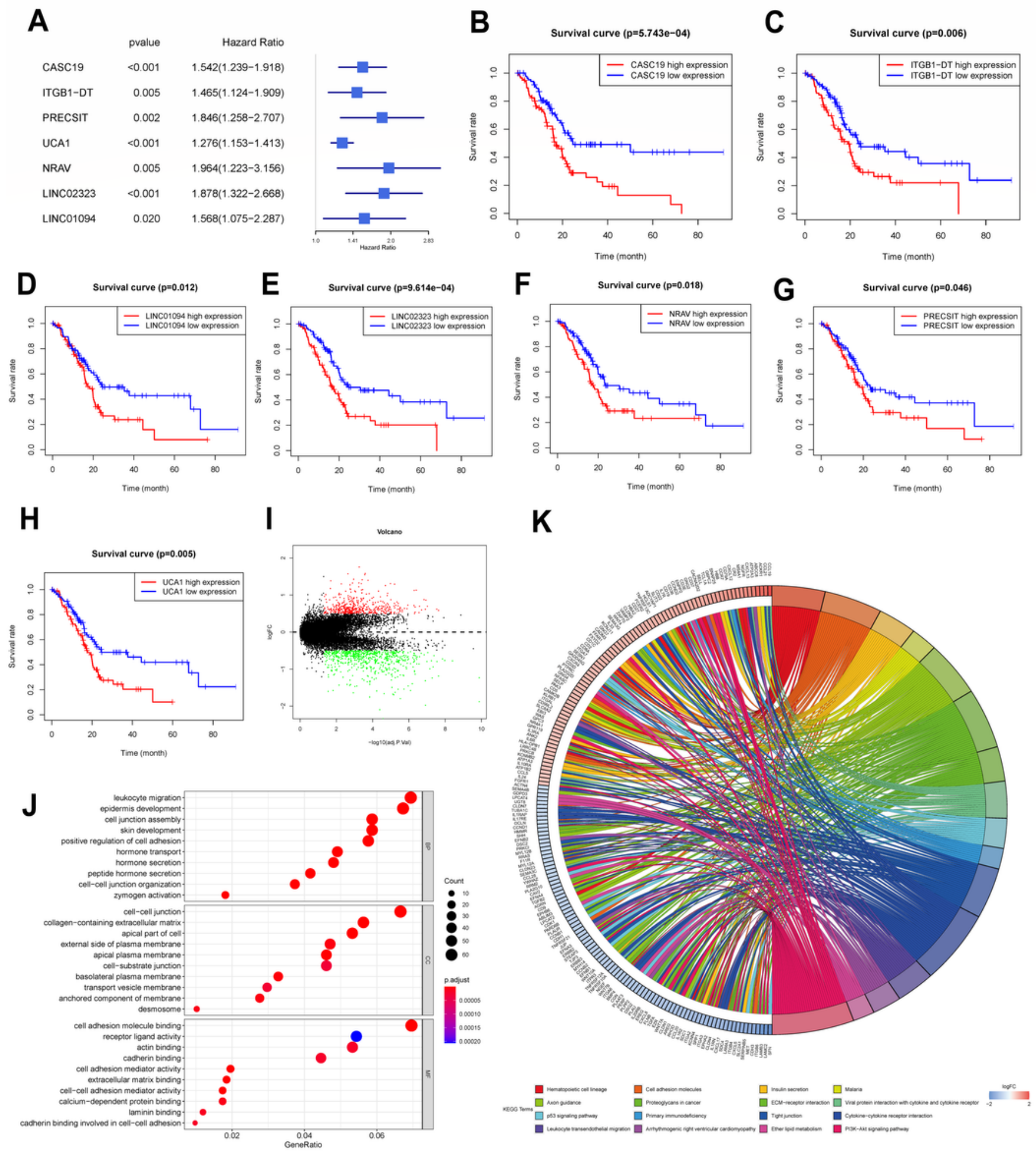

\section{Figure 2}

(A) Forest plot of the prognostic ability of the seven IncRNA-signatures. (B-H) K-M curves were showing that patients with high expression levels of the seven IncRNA-signatures had worse overall survival. (I) 
Differential genes were extracted from patients with different risk subgroups. (J, K) GO (J) and KEGG (K) analyses were performed for different risk subgroups.

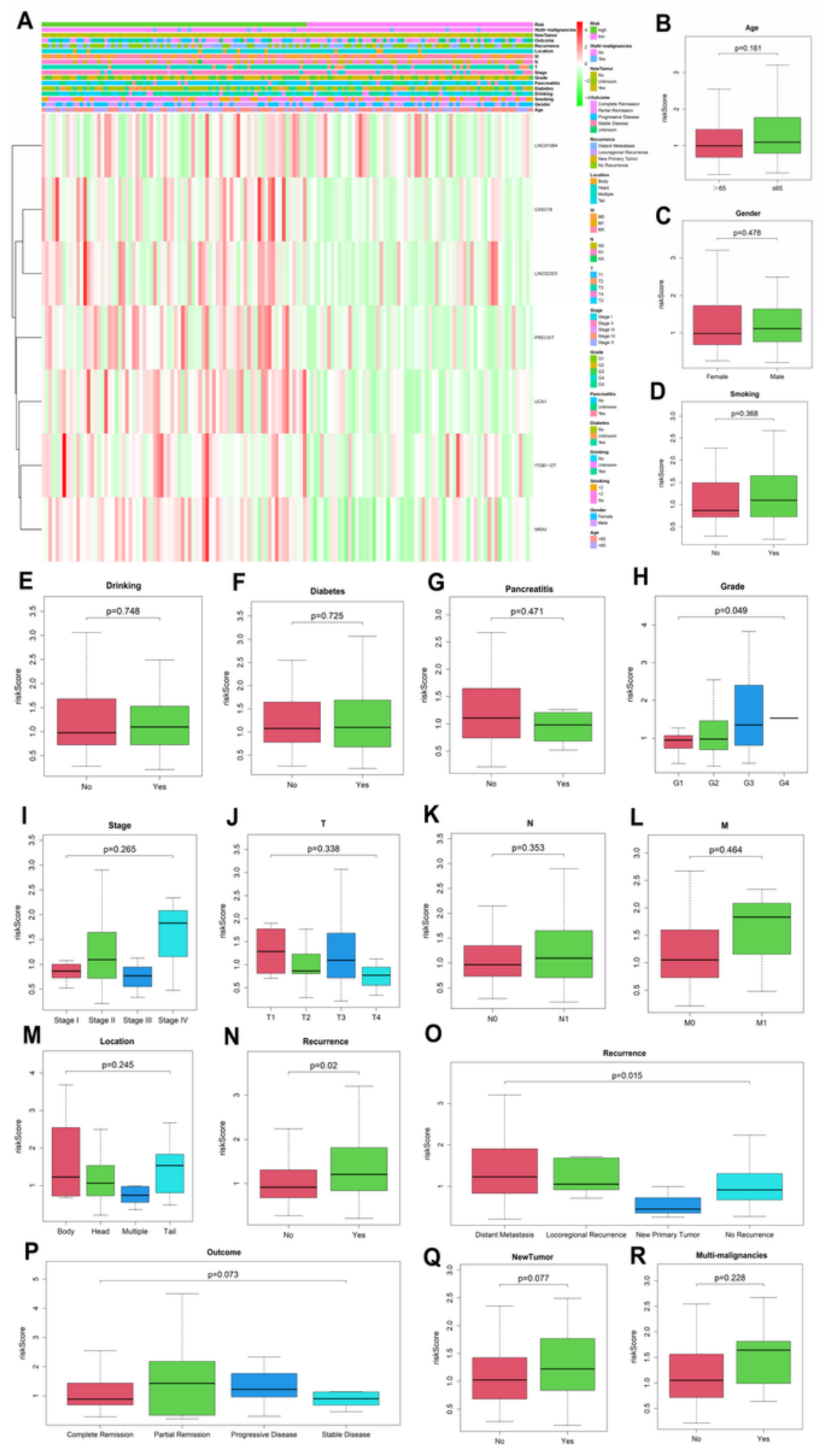

Figure 3

(A) Heatmap of the connections between the expression levels of the seven IncRNA-signatures and clinicopathological features in the TCGA database. (B-R) Patients with different clinicopathological features had different levels of risk scores, calculated based on the seven IncRNA-signatures. 
A

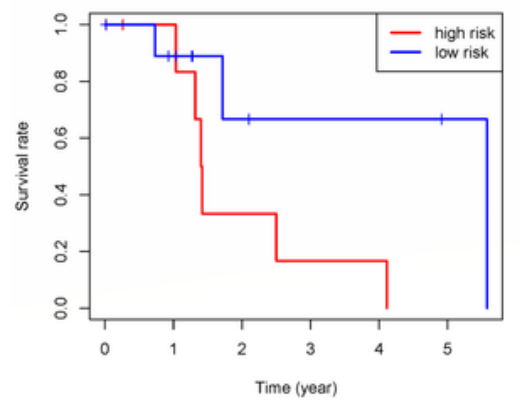

B

G2 ( $p=3.761 e-03)$
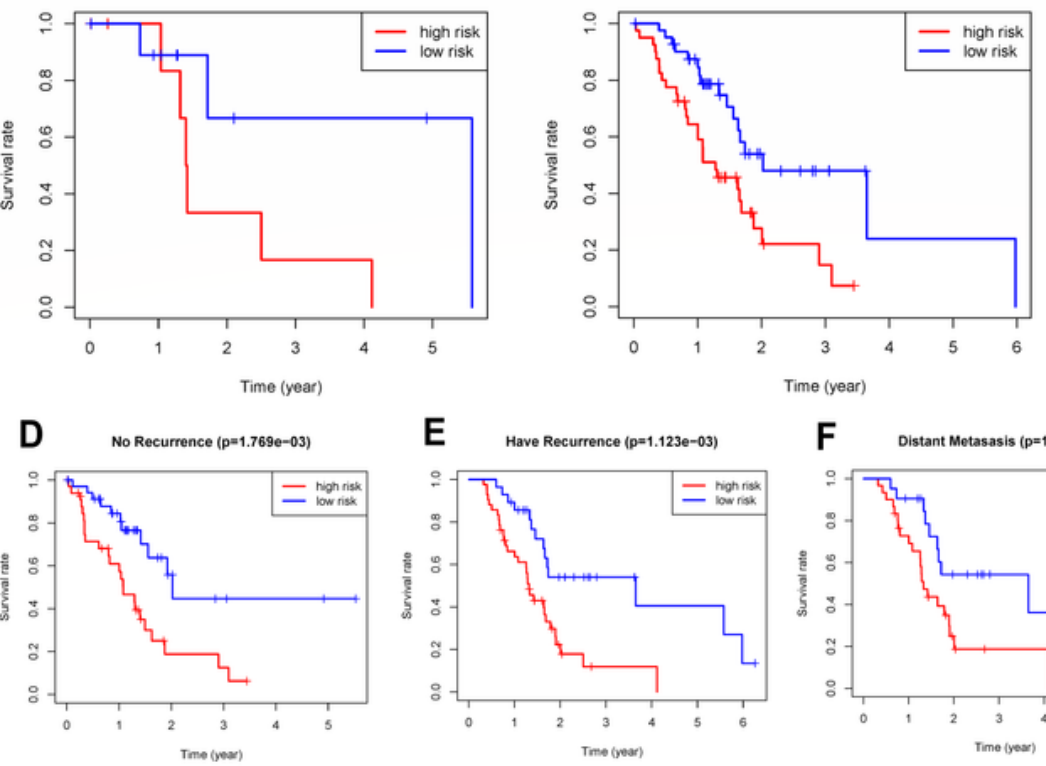

E Have Recurrence (p=1.123e-03)

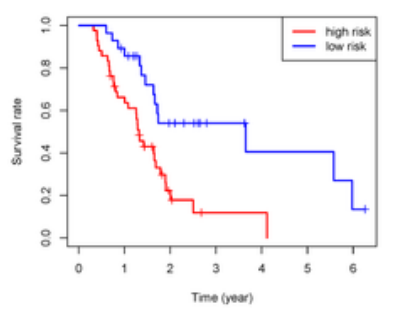

F Distant Metasasis (p=1.3280-02)

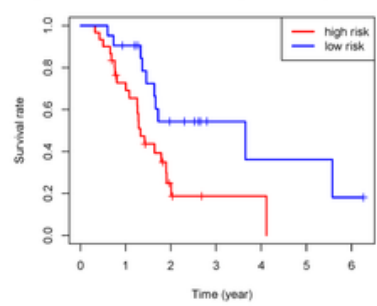

H

Age

Gender

Smoking

Drinking

Diabetes

Pancreatitis

Grade

Stage

$T$

$\mathrm{N}$

M

Location

Recurrence

Outcome

NewTumor

Multi-malignancies

Risk

\section{Pvalue}

Hazard Ratio

1.019(0.997-1.042)

$0.868(0.558-1.353)$

$0.892(0.774-1.029)$

$1.048(0.674-1.631)$

$0.947(0.538-1.668)$

1.343(0.645-2.798)

1.263(0.901-1.770)

1.059(0.634-1.768)

$1.144(0.715-1.831)$

$1.385(0.809-2.369)$

$1.029(0.680-1.558)$

$0.926(0.609-1.408)$

$0.882(0.562-1.385)$

1.591(1.215-2.083)

$0.791(0.507-1.234)$

$1.199(0.432-3.325)$

$2.960(1.807-4.849)$

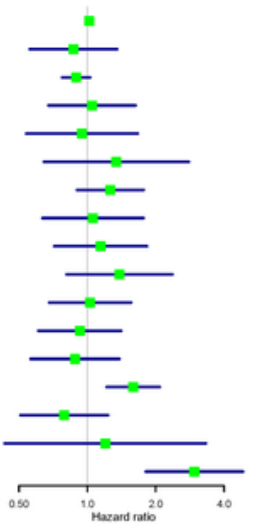

Age

Gender

Smoking

Drinking

Diabetes

Pancreatitis

Grade

Stage

$\mathrm{T}$

$\mathrm{N}$

$M$

Location

Recurrence

Outcome

NewTumor

'Multi-malignancies'

Risk
Hazard Ratio
$1.011(0.987-1.035)$

Pvalue
0.371
0.229
0.035
0.521
0.829
0.446
0.061
0.112
0.559
0.610
0.332
0.568
0.591
0.003
0.105
0.672
$<0.001$

$0.731(0.438-1.219)$

$0.811(0.668-0.986)$

1.181(0.710-1.966)

$0.930(0.483-1.791)$

1.409(0.584-3.398)

1.474(0.982-2.212)

$0.514(0.226-1.167)$

1.242(0.600-2.569)

1.167(0.645-2.110)

$1.290(0.771-2.157)$

$0.871(0.542-1.400)$

$1.238(0.568-2.700)$

$1.584(1.170-2.144)$

$0.519(0.235-1.148)$

$0.786(0.259-2.388)$

2.966(1.687-5.215)
J

C

G3 ( $p=3.419 e-03)$

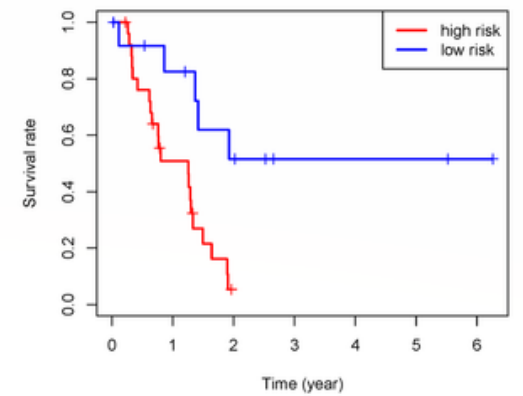

G Locoregional Recurrence ( $p=6.686 \mathrm{e}-02)$
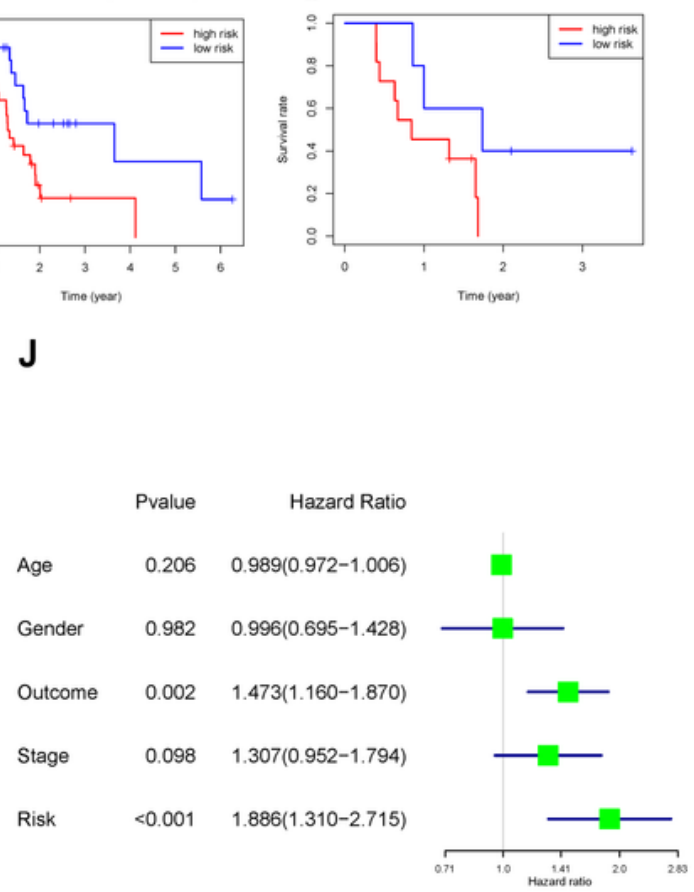

K

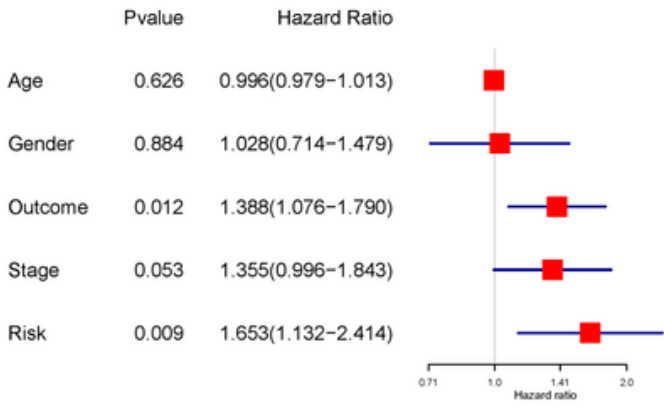

\section{Figure 4}

(A-G) The seven IncRNA-signatures retained its prognostic value in multiple subgroups of PDAC patients, including patients with grade and recurrence. $(\mathrm{H}-\mathrm{K})$ Univariate and multivariate analyses revealed that risk stratification was an independent prognostic predictor in the TCGA $(\mathrm{H}, \mathrm{I})$ and ICGC $(\mathrm{J}, \mathrm{K})$ databases. 

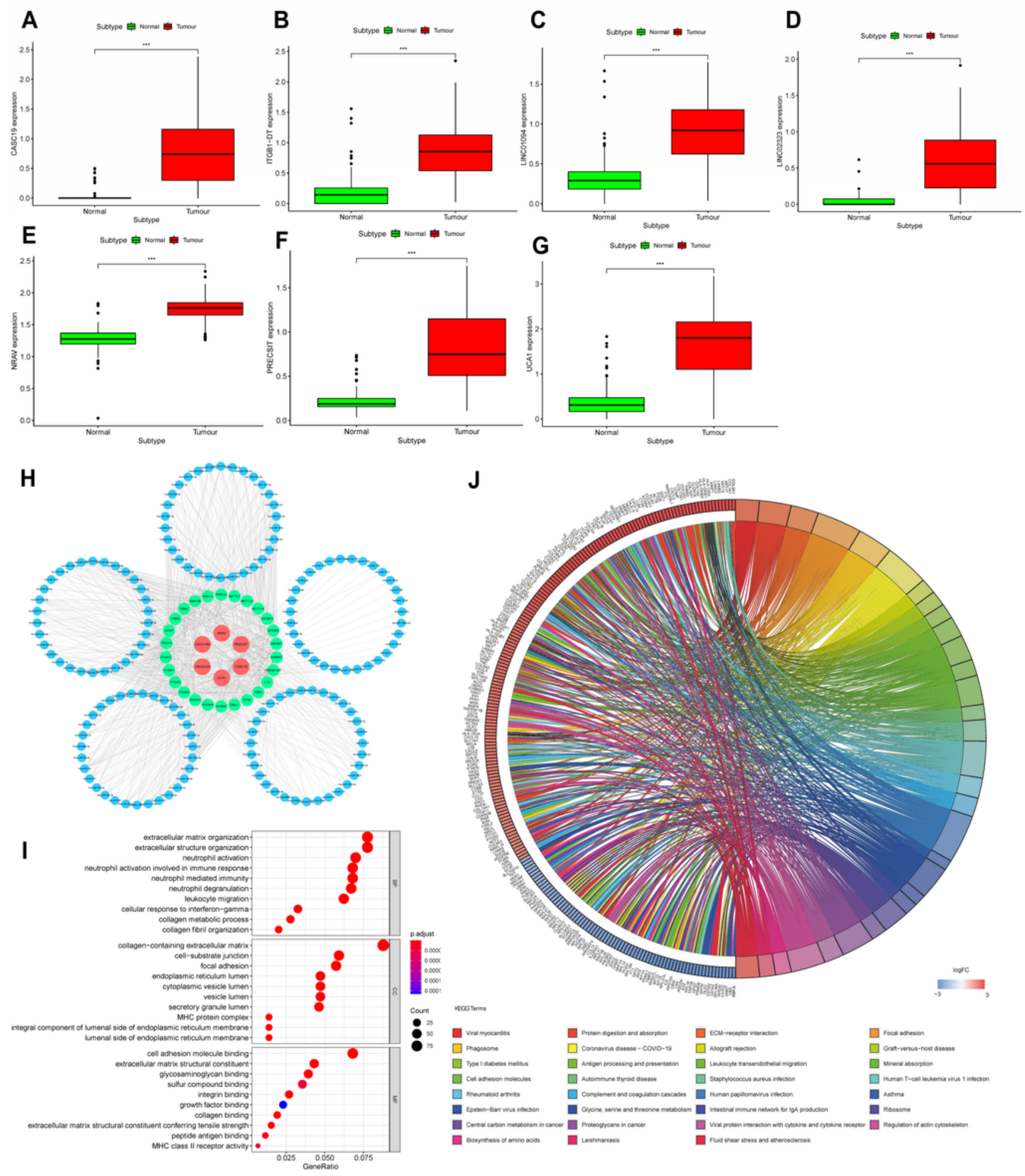

\section{Figure 5}

(A-G) Seven IncRNA-signatures were quantified in tumor and normal tissues using the GTEx-TCGA database. $(\mathrm{H})$ The ceRNA network of the six m6A-related IncRNAs (red) and their target miRNAs (blue) and m6A-related methylation regulative mRNAs (green). (I, J) GO (I) and KEGG (J) analyses were performed for differential target mRNA expression. 